Internat. J. Math. \& Math. Sci.

Vol. 23, No. 11 (2000) 759-776

S0161171200002775

(C) Hindawi Publishing Corp.

\title{
CONDITIONAL GENERALIZED ANALYTIC FEYNMAN INTEGRALS AND A GENERALIZED INTEGRAL EQUATION
}

\author{
SEUNG JUN CHANG, SOON JA KANG, and DAVID SKOUG
}

(Received 5 February 1999)

\begin{abstract}
We use a generalized Brownian motion process to define a generalized Feynman integral and a conditional generalized Feynman integral. We then establish the existence of these integrals for various functionals. Finally we use the conditional generalized Feynman integral to derive a Schrödinger integral equation.
\end{abstract}

Keywords and phrases. Generalized Brownian motion, generalized Feynman integral, conditional generalized Feynman integral, Schrödinger equation.

2000 Mathematics Subject Classification. Primary 28C20.

1. Introduction. Let $L^{2}[0, T]$ denote the space of real valued Lebesgue measurable, square integrable functions on $[0, T]$. Let $C[0, T]$ denote Wiener space, that is, the space of real valued, continuous functions on $[0, T]$ which vanish at 0 .

In various Feynman integration theory the integrand $F$ of the Feynman integral is a functional of the standard Wiener process. In [1], Cameron and Storvick introduced a Banach algebra $S\left(L^{2}[0, T]\right)$ of functionals on Wiener space which are a kind of stochastic Fourier transform of complex Borel measures on $L^{2}[0, T]$. Then they proved the existence of the analytic Feynman integral for functionals in $S\left(L^{2}[0, T]\right)$. In [4], Chung and Skoug introduced the concept of a conditional Feynman integral of a functional on Wiener space given a functional $X$ and established the existence of the conditional analytic Feynman integral for all functionals in the Banach algebra $S\left(L^{2}[0, T]\right)$. In this paper we extend the results involving the analytic Feynman and conditional analytic Feynman integrals of functionals of the standard Wiener process to those of functionals involving a more general stochastic process. We note that the Wiener process is free of drift and is stationary in time. However, the stochastic process considered in this paper is a process subject to drift and is nonstationary in time. We also use this conditional generalized analytic Feynman integral to provide a solution to an integral equation formally equivalent to the generalized Schrödinger equation.

2. Definitions and preliminaries. Let $D=[0, T]$ and let $(\Omega, \mathscr{B}, P)$ be a probability measure space. A real valued stochastic process $Y$ on $(\Omega, \mathscr{B}, P)$ and $D$ is called a generalized Brownian motion process if $Y(0, \omega)=0$ a.e. and for $0 \leq t_{0}<t_{1}<\cdots<t_{n} \leq T$, the $n$-dimensional random vector $\left(Y\left(t_{1}, \omega\right), \ldots, Y\left(t_{n}, \omega\right)\right)$ is normally distributed with the density function 


$$
\begin{aligned}
K(\vec{t}, \vec{\eta})= & \left((2 \pi)^{n} \prod_{j=1}^{n}\left(b\left(t_{j}\right)-b\left(t_{j-1}\right)\right)\right)^{-1 / 2} \\
& \cdot \exp \left\{-\frac{1}{2} \sum_{j=1}^{n}=\frac{\left(\left(\eta_{j}-a\left(t_{j}\right)\right)-\left(\eta_{j-1}-a\left(t_{j-1}\right)\right)\right)^{2}}{b\left(t_{j}\right)-b\left(t_{j-1}\right)}\right\}
\end{aligned}
$$

where $\vec{\eta}=\left(\eta_{1}, \ldots, \eta_{n}\right), \eta_{0}=0, \vec{t}=\left(t_{1}, \ldots, t_{n}\right), t_{0}=0$, and $a(t)$ is a continuous realvalued function of bounded variation with $a(0)=0$, and $b(t)$ is a strictly increasing, continuous real-valued function with $b(0)=0$.

As explained in [9, pages 18-20], $Y$ induces a probability measure $\mu$ on the measurable space $\left(\mathbb{R}^{D}, \mathscr{P}^{D}\right)$ where $\mathbb{R}^{D}$ is the space of all real-valued functions $x(t), t \in D$, and $\mathscr{B}^{D}$ is the smallest $\sigma$-algebra of subsets of $\mathbb{R}^{D}$ with respect to which all the coordinate evaluation maps $e_{t}(x)=x(t)$ defined on $\mathbb{R}^{D}$ are measurable. The triple $\left(\mathbb{R}^{D}, \mathscr{B}^{D}, \mu\right)$ is a probability measure space.

We note that the generalized Brownian motion process $Y$ determined by $a(\cdot)$ and $b(\cdot)$ is a Gaussian process with mean function $a(t)$ and covariance function $r(s, t)=$ $\min \{b(s), b(t)\}$. By [9, Theorem 14.2, page 187], the probability measure $\mu$ induced by $Y$, taking a separable version, is supported by $C_{a, b}[0, T]$ (which is equivalent to the Banach space of continuous functions $x$ on $[0, T]$ with $\mathrm{x}(0)=0$ under the sup norm). Hence $\left(C_{a, b}[0, T], \mathscr{B}\left(C_{a, b}[0, T]\right), \mu\right)$ is the function space induced by $Y$ where $\mathscr{B}\left(C_{a, b}[0, T]\right)$ is the Borel $\sigma$-algebra of $C_{a, b}[0, T]$. Note that we can also express $x$ in the form

$$
x(t)=w(b(t))+a(t), \quad 0 \leq t \leq T
$$

where $w(\cdot)$ is the standard Brownian motion process.

A subset $B$ of $C_{a, b}[0, T]$ is said to be scale-invariant measurable [2,5] provided $\rho B$ is $\mathscr{B}\left(C_{a, b}[0, T]\right)$-measurable for all $\rho>0$, and a scale-invariant measurable set $N$ is said to be scale-invariant null set provided $\mu(\rho N)=0$ for all $\rho>0$. A property that holds except on a scale-invariant null set is said to hold scale-invariant almost everywhere (s-a.e.).

Let $L_{a, b}^{2}[0, T]$ be the Hilbert space of functionals on $[0, T]$ which are Lebesgue measurable and square integrable with respect to the Lebesgue Stieltjes measures on $[0, T]$ induced by $a(\cdot)$ and $b(\cdot)$; i.e.,

$$
L_{a, b}^{2}[0, T]=\left\{v: \int_{0}^{T} v^{2}(s) d b(s)<\infty \text { and } \int_{0}^{T} v^{2}(s) d|a|(s)<\infty\right\}
$$

where $|a|$ denotes total variation. For $h \in L_{a, b}^{2}[0, T]$ with $\|h\|>0$, let $z(x, t)$ denote the Paley-Wiener-Zygmund (PWZ) stochastic integral

$$
z(x, t)=\int_{0}^{t} h(s) d x(s),
$$

let $\beta(t)=\int_{0}^{t} h^{2}(s) d b(s)$, and let $\alpha(t)=\int_{0}^{t} h(s) d a(s)$. Then $z$ is a Gaussian process with mean

$$
E_{x}[z(x, t)]=\int_{0}^{t} h(u) d a(u)=\alpha(t)
$$


and covariance function

$$
E_{x}[(z(x, s)-\alpha(s))(z(x, t)-\alpha(t))]=\int_{0}^{s \wedge t} h^{2}(u) d b(u)=\beta(s \wedge t)
$$

where $s \wedge t$ is the minimum of $s$ and $t$. Of course if $h(t) \equiv 1, a(t) \equiv 0$, and $b(t)=t$ on $[0, T]$, then the Gaussian process (2.4) is an ordinary Wiener process.

We denote the function space integral of a $\mathscr{B}\left(C_{a, b}[0, T]\right)$ - measurable function $F$ by

$$
E[F]=\int_{C_{a, b}[0, T]} F(x) d \mu(x)
$$

whenever the integral exists.

First we state the definition of the conditional function space integral [4, 8].

DEFINITION 2.1. Let $X$ be a real-valued measurable function on $C_{a, b}[0, T]$ whose probability distribution function $\mu_{X}$ is absolutely continuous with respect to Lebesgue measure on $\mathbb{R}$. Let $F$ be a complex-valued $\mu$-integrable function on $C_{a, b}[0, T]$. Then the conditional integral of $F$ given $X$, denoted by $E[F \mid X](\eta)$, is a Lebesgue measurable function of $\eta$, unique up to null sets in $\mathbb{R}$, satisfying the equation

$$
\int_{X^{-1}(B)} F(x) d \mu(x)=\int_{B} E[F \mid X](\eta) d \mu_{X}(\eta)
$$

for all Borel sets $B$ in $\mathbb{R}$.

We are now ready to state the definitions of the generalized analytic Feynman integral and the conditional generalized analytic Feynman integral [4, 3].

DeFinITION 2.2. Let $\mathbb{C}$ denote the complex numbers. Let $\mathbb{C}_{+}=\{\lambda \in \mathbb{C}: \operatorname{Re} \lambda>0\}$ and $\tilde{\mathbb{C}}_{+}=\{\lambda \in \mathbb{C}: \lambda \neq 0$ and $\operatorname{Re} \lambda \geq 0\}$. Let $F: C_{a, b}[0, T] \rightarrow \mathbb{C}$ be such that for each $\lambda>0$, the function space integral

$$
J(\lambda)=\int_{C_{a, b}[0, T]} F\left(\lambda^{-1 / 2} z(x, \cdot)\right) d \mu(x)=E_{x}\left[F\left(\lambda^{-1 / 2} z(x, \cdot)\right)\right]
$$

exists for all $\lambda>0$. If there exists a function $J^{*}(\lambda)$ analytic in $\mathbb{C}_{+}$such that $J^{*}(\lambda)=J(\lambda)$ for all $\lambda>0$, then $J^{*}(\lambda)$ is defined to be the analytic function space integral of $F$ over $C_{a, b}[0, T]$ with parameter $\lambda$, and for $\lambda \in \mathbb{C}_{+}$we write

$$
E^{a n_{\lambda}}[F] \equiv E_{x}^{a n_{\lambda}}[F(z(x, \cdot))]=J^{*}(\lambda)
$$

Let $q \neq 0$ be a real number and let $F$ be a function such that $E^{a n_{\lambda}}[F]$ exists for all $\lambda \in \mathbb{C}_{+}$. If the following limit exists, we call it the generalized analytic Feynman integral of $F$ with parameter $q$ and we write

$$
E^{a n f_{q}}[F] \equiv E_{x}^{a n f_{q}}[F(z(x, \cdot))]=\lim _{\lambda \rightarrow-i q} E^{a n_{\lambda}}[F]
$$

where $\lambda$ approaches $-i q$ through $\mathbb{C}_{+}$. 
DEFINITION 2.3. Let $F: C_{a, b}[0, T] \rightarrow \mathbb{C}$ be such that for each $\lambda>0$,

$$
\int_{C_{a, b}[0, T]}\left|F\left(\lambda^{-1 / 2} z(x, \cdot)\right)\right| d \mu(x)<\infty
$$

Let $X: C_{a, b}[0, T] \rightarrow \mathbb{R}$ be a scale-invariant measurable function. For $\lambda>0$, let

$$
J_{\lambda}(\eta)=E_{x}\left[F\left(\lambda^{-1 / 2} z(x, \cdot)\right) \mid X\left(\lambda^{-1 / 2} x\right)\right](\eta)
$$

denote the conditional function space integral of $F\left(\lambda^{-1 / 2} z(x, \cdot)\right)$ given $X\left(\lambda^{-1 / 2} x\right)$. If for a.e. $\eta \in \mathbb{R}$, there exists a function $J_{\lambda}^{*}(\eta)$ analytic in $\lambda$ on $\mathbb{C}_{+}$such that $J_{\lambda}^{*}(\eta)=J_{\lambda}(\eta)$ for all $\lambda>0$, then $J_{\lambda}^{*}(\cdot)$ is defined to be the conditional analytic function space integral of $F$ given $X$ with parameter $\lambda$ and we write

$$
E^{a n_{\lambda}}[F \mid X](\eta) \equiv E_{x}^{a n_{\lambda}}[F(z(x, \cdot)) \mid X(x)](\eta)=J_{\lambda}^{*}(\eta)
$$

If for fixed real $q \neq 0$ the limit

$$
\lim _{\lambda \rightarrow-i q} E^{a n_{\lambda}}[F \mid X](\eta)
$$

exists for a.e. $\eta \in \mathbb{R}$, where $\lambda \rightarrow-i q$ through $\mathbb{C}_{+}$, we will denote the value of this limit by $E_{x}^{a n f_{q}}[F \mid X](\eta)$ and call it the conditional generalized analytic Feynman integral of $F$ given $X$ with parameter $q$.

REMARK 2.4. In [8], Park and Skoug gave a formula for expressing conditional Wiener integrals in terms of ordinary Wiener integrals; namely that for $\lambda>0$,

$$
\begin{aligned}
E_{x}\left[F\left(\lambda^{-1 / 2} z(x, \cdot)\right) \mid\right. & \left.\lambda^{-1 / 2} z(x, T)\right](\eta) \\
& =E_{x}\left[F\left(\lambda^{-1 / 2} z(x, \cdot)-\lambda^{-1 / 2} \frac{\beta(\cdot)}{\beta(T)} z(x, T)+\frac{\beta(\cdot)}{\beta(T)} \eta\right)\right]
\end{aligned}
$$

where $\beta(t)=\int_{0}^{t} h^{2}(s) d b(s)$. Thus we have that

$$
\begin{gathered}
E_{x}^{a n_{\lambda}}[F(z(x, \cdot)) \mid z(x, T)](\eta)=E_{x}^{a n_{\lambda}}\left[F\left(z(x, \cdot)-\frac{\beta(\cdot)}{\beta(T)} z(x, T)+\frac{\beta(\cdot)}{\beta(T)} \eta\right)\right] \\
E_{x}^{a n f_{q}}[F(z(x, \cdot)) \mid z(x, T)](\eta)=E_{x}^{a n f_{q}}\left[F\left(z(x, \cdot)-\frac{\beta(\cdot)}{\beta(T)} z(x, T)+\frac{\beta(\cdot)}{\beta(T)} \eta\right)\right]
\end{gathered}
$$

where in (2.17) and (2.18)the existence of either side implies the existence of the other side and their equality.

The following function space integral formula is used throughout this paper:

$$
E_{x}\left[\exp \left\{i \lambda^{-1 / 2}\langle v, x\rangle\right\}\right]=\exp \left\{-\frac{1}{2 \lambda}\left(v^{2}, b\right)+i \lambda^{-1 / 2}(v, a)\right\}
$$


for $\lambda>0, v \in L_{a, b}^{2}[0, T]$, and where $\langle v, x\rangle$ denotes the PWZ stochastic integral $\int_{0}^{T} v(s) d x(s)$ and $\left(v^{2}, b\right)$ denotes the Lebesgue Stieltjes integral $\int_{0}^{T} v^{2}(s) d b(s)$.

3. Generalized analytic Feynman integrals. Let $M\left(L_{a, b}^{2}[0, T]\right)$ be the space of $\mathbb{C}$ valued, countably additive finite Borel measures on $L_{a, b}^{2}[0, T]$. The Banach algebra $S\left(L_{a, b}^{2}[0, T]\right)$ consists of functionals $F$ on $C_{a, b}[0, T]$ expressible in the form

$$
F(x)=\int_{L_{a, b}^{2}[0, T]} \exp \{i\langle v, x\rangle\} d \sigma(v)
$$

for s-a.e. $x \in C_{a, b}[0, T]$ where $\sigma$ is an element of $M\left(L_{a, b}^{2}[0, T]\right)$. Further works on $S\left(L_{a, b}^{2}[0, T]\right)$ shows that it contains many functionals of interest in Feynman integration theory $[1,3,4,6,7]$.

REMARK 3.1. (i) Throughout the remainder of this paper, since we are usually considering functionals in the Banach algebra $S\left(L_{a, b}^{2}[0, T]\right)$, we will always assume that $h$ is an element of $L_{\infty}[0, T]$ with $\|h\|>0$. This will insure that the various Lebesgue Stieltjes integrals that arise will exist. For example, the Lebesgue Stieltjes integrals $\left(v^{2} h^{2}, b\right),\left(v h^{2}, b\right)$, and $(v h, a)$ will exist for all $v \in L_{a, b}^{2}[0, T]$.

(ii) Note that for $F \in S\left(L_{a, b}^{2}[0, T]\right)$, the function $G: C_{a, b}[0, T] \rightarrow \mathbb{C}$ given by

$$
G(x)=F(z(x, \cdot))
$$

with $h \in L_{\infty}[0, T]$, belongs to the Banach algebra $S\left(L_{a, b}^{2}[0, T]\right)[3]$.

The following lemma follows easily from the definition of the PWZ stochastic integral.

LEMMA 3.2 [3]. For each $v \in L_{a, b}^{2}[0, T]$ and each $h \in L_{\infty}[0, T]$,

$$
\int_{0}^{T} v(s) d z(x, s) \equiv \int_{0}^{T} v(s) d\left[\int_{0}^{s} h(u) d x(u)\right]=\langle v h, x\rangle
$$

for s-a.e. $x \in C_{a, b}[0, T]$.

THEOREM 3.3. Let $F \in S\left(L_{a, b}^{2}[0, T]\right)$ be given by (3.1) and let $z(x, t)$ be given by (2.4) with $h \in L_{\infty}[0, T]$. Then for each $\lambda \in \mathbb{C}_{+}$, the analytic function space integral $E^{a n_{\lambda}}[F]$ exists and is given by the formula

$$
E^{a n_{\lambda}}[F]=\int_{L_{a, b}^{2}[0, T]} \exp \left\{-\frac{1}{2 \lambda}\left(v^{2} h^{2}, b\right)+i \lambda^{-1 / 2}(v h, a)\right\} d \sigma(v) .
$$

Furthermore, the generalized analytic Feynman integral $E^{a n f_{a}}[F]$ exists for all real $q \neq 0$ and is given by the formula

$$
E^{a n f_{q}[F]}=\int_{L_{a, b}^{2}[0, T]} \exp \left\{-\frac{i}{2 q}\left(v^{2} h^{2}, b\right)+i\left(\frac{i}{q}\right)^{1 / 2}(v h, a)\right\} d \sigma(v) .
$$


Proof. By (2.10), the Fubini theorem, Lemma 3.2 and (2.19), we have for all $\lambda>0$,

$$
\begin{aligned}
E^{a n_{\lambda}[F]} & =\int_{C_{a, b}[0, T]} F\left(\lambda^{-1 / 2} z(x, \cdot)\right) d \mu(x) \\
& =\int_{L_{a, b}^{2}[0, T]} \int_{C_{a, b}[0, T]} \exp \left\{i \lambda^{-1 / 2}\langle v, z(x, \cdot)\rangle\right\} d \mu(x) d \sigma(v) \\
& =\int_{L_{a, b}^{2}[0, T]} \int_{C_{a, b}[0, T]} \exp \left\{i \lambda^{-1 / 2}\langle v h, x\rangle\right\} d \mu(x) d \sigma(v) \\
& =\int_{L_{a, b}^{2}[0, T]} \exp \left\{-\frac{1}{2 \lambda}\left(v^{2} h^{2}, b\right)+i \lambda^{-1 / 2}(v h, a)\right\} d \sigma(v) .
\end{aligned}
$$

But (3.6) is an analytic function of $\lambda$ throughout $\mathbb{C}_{+}$, and is a continuous function of $\lambda$ in $\tilde{\mathbb{C}}_{+}$since $\sigma$ is a finite Borel measure. Thus equations (3.4) and (3.5) are established.

COROLLARY 3.4 [1]. In Theorem 3.3, if $a(t) \equiv 0, b(t)=t$, and $h(t) \equiv 1$, then $L_{a, b}^{2}[0, T]=$ $L^{2}[0, T]$ and the generalized analytic Feynman integral $E^{a n f_{a}}[F]$ is an ordinary analytic Feynman integral and

$$
E^{a n f_{q}}[F]=\int_{L^{2}[0, T]} \exp \left\{-\frac{i}{2 q} \int_{0}^{T} v^{2}(s) d s\right\} d \sigma(v) .
$$

4. Conditional generalized analytic Feynman integrals. Throughout this section we will condition by the function $X: C_{a, b}[0, T] \rightarrow \mathbb{R}$ given by

$$
X\left(\lambda^{-1 / 2} x\right) \equiv \lambda^{-1 / 2} z(x, T)=\lambda^{-1 / 2}\langle h, x\rangle
$$

for s-a.e. $x$ on $C_{a, b}[0, T]$. In Theorem 4.1, for $F \in S\left(L_{a, b}^{2}[0, T]\right)$, we evaluate the conditional generalized Feynman integral $E^{\operatorname{anf}_{q}}[F \mid X]$.

THEOREM 4.1. Let $F \in S\left(L_{a, b}^{2}[0, T]\right)$ be given by (3.1), let $X$ be given by (4.1) with $h \in L_{\infty}[0, T]$, and let $\beta(t)=\int_{0}^{t} h^{2}(s) d b(s)$. Then the conditional function space integral $E^{a n_{\lambda}}[F \mid X]$ exists and is given by the formula

$$
\begin{aligned}
& E^{a n_{\lambda}}[F \mid X](\eta)=\int_{L_{a, b}^{2}[0, T]} \exp \left\{\frac{i \eta}{\beta(T)}\left(v h^{2}, b\right)-\frac{1}{2 \lambda}\left(\left[v-\frac{\left(v h^{2}, b\right)}{\beta(T)}\right]^{2} h^{2}, b\right)\right. \\
& \left.+i \lambda^{-1 / 2}\left(\left[v-\frac{\left(v h^{2}, b\right)}{\beta(T)}\right] h, a\right)\right\} d \sigma(v)
\end{aligned}
$$

for all $\lambda \in \mathbb{C}_{+}$. Furthermore, the conditional generalized analytic Feynman integral $E^{a n} f_{a}[F \mid X]$ exists for all real $q \neq 0$ and is given by the formula

$$
\begin{aligned}
& E^{a n f_{a}}[F \mid X](\eta)=\int_{L_{a, b}^{2}[0, T]} \exp \left\{\frac{i \eta}{\beta(T)}\left(v h^{2}, b\right)-\frac{i}{2 q}\left(\left[v-\frac{\left(v h^{2}, b\right)}{\beta(T)}\right]^{2} h^{2}, b\right)\right. \\
& \left.+i\left(\frac{i}{q}\right)^{1 / 2}\left(\left[v-\frac{\left(v h^{2}, b\right)}{\beta(T)}\right] h, a\right)\right\} d \sigma(v) .
\end{aligned}
$$


Proof. By the definition of $S\left(L_{a, b}^{2}[0, T]\right)$, we have that for all $\lambda>0$,

$$
F\left(\lambda^{-1 / 2} z(x, \cdot)\right)=\int_{L_{a, b}^{2}[0, T]} \exp \left\{i \int_{0}^{T} v(s) d\left(\lambda^{-1 / 2} z(x, s)\right)\right\} d \sigma(v)
$$

for s-a.e. $x \in C_{a, b}[0, T]$. Now using (2.14), (2.17), Lemma 3.2, the Fubini theorem, and (2.19), we obtain the formula

$$
\begin{aligned}
E^{a n_{\lambda}} & {[F \mid X](\eta) } \\
& =E_{x}\left[F\left(\lambda^{-1 / 2} z(x, \cdot)\right) \mid \lambda^{-1 / 2} z(x, T)\right](\eta) \\
& =E_{x}\left[\int_{L_{a, b}^{2}[0, T]} \exp \left\{i \int_{0}^{T} v(s) d\left(\lambda^{-1 / 2} z(x, s)\right)\right\} d \sigma(v) \mid \lambda^{-1 / 2} z(x, T)=\eta\right] \\
& =\int_{C_{a, b}[0, T]} \int_{L_{a, b}^{2}[0, T]} \exp \left\{i \int_{0}^{T} v(s) d\left(\lambda^{-1 / 2}\left(z(x, s)-\frac{\beta(s)}{\beta(T)} z(x, T)\right)+\frac{\beta(s)}{\beta(T)} \eta\right)\right\} \\
& =\int_{L_{a, b}^{2}[0, T]} \exp \left\{\frac{i \eta}{\beta(T)}\left(v h^{2}, b\right)\right\} \\
& =\int_{L_{a, b}^{2}[0, T]} \exp \left\{\frac{i \eta}{\beta(T)}\left(v h^{2}, b\right)-\frac{1}{2 \lambda}\left(\left[v-\frac{\left(v h^{2}, b\right)}{\beta(T)}\right]^{2} h^{2}, b\right)\right. \\
& \left.+i \lambda^{-1 / 2}\left(\left[v-\frac{\left(v h^{2}, b\right)}{\beta(T)}\right] h, a\right)\right\} d \sigma(v)
\end{aligned}
$$

for all $(\lambda, \eta) \in(0, \infty) \times \mathbb{R}$. Hence, since $\sigma$ is a finite Borel measure on $L_{a, b}^{2}[0, T]$, the last expression on the right-hand side of (4.5) is an analytic function of $\lambda$ throughout $\mathbb{C}_{+}$, and is a continuous function of $\lambda$ in $\tilde{\mathbb{C}}_{+}$. Thus, (4.2) and (4.3) are established.

COROLLARY 4.2 [1]. In Theorem 4.1, if $a(t) \equiv 0, b(t)=t$, and $h(t) \equiv 1$, then the conditional generalized analytic Feynman integral $E^{a n f_{a}}[F \mid X]$ is an ordinary conditional Feynman integral and

$E^{a n f_{q}}[F \mid X](\eta)=\int_{L^{2}[0, T]} \exp \left\{\frac{i \eta}{T} \int_{0}^{T} v(s) d s-\frac{i}{2 q} \int_{0}^{T} v^{2}(s) d s+\frac{i}{2 q T}\left(\int_{0}^{T} v(s) d s\right)^{2}\right\} d \sigma(v)$.

In our next theorem we show that if we multiply $E^{a n f_{q}}[F \mid X](\eta)$ by $(q / 2 \pi i \beta(T))^{1 / 2}$ $\cdot \exp \left\{(i q / 2 \beta(T))\left(\eta-(i / q)^{1 / 2} \alpha(T)\right)^{2}\right\}$, the analytic extension of the Radon-Nykodym derivative evaluated at $\lambda=-i q$, and then integrate over $\mathbb{R}$ we obtain the generalized analytic Feynman integral $E^{a n f_{q}}[F]$ where $\alpha(T)=(h, a)$ is the mean of $z(x, T)$. To do so we need the following summation procedure. Let 


$$
\int_{\mathbb{R}} f(\eta) d \eta \equiv \lim _{A \rightarrow+\infty} \int_{\mathbb{R}} f(\eta) \exp \left\{-\frac{\eta^{2}}{2 A}\right\} d \eta
$$

whenever the expression on the right-hand side exists. But if $f \in L^{1}(\mathbb{R})$, it is clear by the dominated convergence theorem that

$$
\int_{\mathbb{R}} f(\eta) d \eta=\int_{\mathbb{R}} f(\eta) d \eta
$$

THEOREM 4.3. Let $F \in S\left(L_{a, b}^{2}[0, T]\right)$ be given by (3.1) and let $X$ be given by (4.1) with $h \in L_{\infty}[0, T]$. Then for all $\lambda \in \mathbb{C}_{+}$,

$$
E^{a n_{\lambda}}[F]=\int_{\mathbb{R}}\left(\frac{\lambda}{2 \pi \beta(T)}\right)^{1 / 2} \exp \left\{-\frac{\lambda\left(\eta-\lambda^{-1 / 2} \alpha(T)\right)^{2}}{2 \beta(T)}\right\} E^{a n_{\lambda}}[F \mid X](\eta) d \eta
$$

and for all real $q \neq 0$,

$$
\begin{aligned}
& E^{a n f_{q}[F]} \\
& \quad=\int_{\mathbb{R}}\left(\frac{q}{2 \pi i \beta(T)}\right)^{1 / 2} \exp \left\{\frac{i q\left(\eta-(i / q)^{1 / 2} \alpha(T)\right)^{2}}{2 \beta(T)}\right\} E^{a n f_{q}}[F \mid X](\eta) d \eta .
\end{aligned}
$$

Proof. We will obtain the formula (4.10). The proof of (4.9) is similar to (4.10) but easier since the summation procedure is not needed. Let $q \neq 0$ be given. By using (2.15), (2.19), (4.3), (4.7), the Fubini theorem, and (3.5), we obtain that

$$
\begin{gathered}
\int_{\mathbb{R}}\left(\frac{q}{2 \pi i \beta(T)}\right)^{1 / 2} \exp \left\{\frac{i q\left(\eta-(i / q)^{1 / 2} \alpha(T)\right)^{2}}{2 \beta(T)}\right\} E^{a n f_{q}}[F \mid X](\eta) d \eta \\
=\lim _{A \rightarrow+\infty} \int_{\mathbb{R}}\left(\frac{q}{2 \pi i \beta(T)}\right)^{1 / 2} \exp \left\{\frac{i q\left(\eta-(i / q)^{1 / 2} \alpha(T)\right)^{2}}{2 \beta(T)}-\frac{\eta^{2}}{2 A}\right\} \\
\cdot\left[\int _ { L _ { a , b } ^ { 2 } [ 0 , T ] } \operatorname { e x p } \left\{\frac{i \eta}{\beta(T)}\left(v h^{2}, b\right)-\frac{i}{2 q}\left(\left[v-\frac{\left(v h^{2}, b\right)}{\beta(T)}\right]^{2} h^{2}, b\right)\right.\right. \\
\left.\left.+i\left(\frac{i}{q}\right)^{1 / 2}\left(\left[v-\frac{\left(v h^{2}, b\right)}{\beta(T)}\right] h, a\right)\right\} d \sigma(v)\right] d \eta \\
\lim _{A \rightarrow+\infty} \int_{L_{a, b}^{2}[0, T]} \exp \left\{-\frac{\alpha^{2}(T)}{2 \beta(T)}-\frac{i}{2 q}\left(\left[v-\frac{\left(v h^{2}, b\right)}{\beta(T)}\right]^{2} h^{2}, b\right)\right. \\
\left.\quad+i\left(\frac{i}{q}\right)^{1 / 2}\left(\left[v-\frac{\left(v h^{2}, b\right)}{\beta(T)}\right] h, a\right)\right\} \\
\quad\left(\frac{q}{2 \pi i \beta(T)}\right)^{1 / 2}\left[\int_{\mathbb{R}} \exp \left\{\frac{i q \eta^{2}-2 q(i / q)^{1 / 2} \alpha(T) \eta}{2 \beta(T)}-\frac{\eta^{2}}{2 A}+\frac{i\left(v h^{2}, b\right) \eta}{\beta(T)}\right\} d \eta\right] d \sigma(v)
\end{gathered}
$$




$$
\begin{aligned}
& =\lim _{A \rightarrow+\infty} \int_{L_{a, b}^{2}[0, T]}\left(\frac{q}{2 \pi \beta(T)}\right)^{1 / 2}\left(\frac{2 \pi A \beta(T)}{\beta(T)-i q A}\right)^{1 / 2} \\
& \cdot \exp \left\{-\frac{\alpha^{2}(T)}{2 \beta(T)}-\frac{i}{2 q}\left(\left[v-\frac{\left(v h^{2}, b\right)}{\beta(T)}\right]^{2} h^{2}, b\right)\right. \\
& +i\left(\frac{i}{q}\right)^{1 / 2}\left(\left[v-\frac{\left(v h^{2}, b\right)}{\beta(T)}\right] h, a\right) \\
& \left.-\frac{A\left(q(i / q)^{1 / 2} \alpha(T)-\left(v h^{2}, b\right)\right)^{2}}{2 \beta(T)(\beta(T)-A i q)}\right\} d \sigma(v) \\
& =\int_{L_{a, b}^{2}[0, T]} \exp \left\{-\frac{\alpha^{2}(T)}{2 \beta(T)}-\frac{i}{2 q}\left(\left[v-\frac{\left(v h^{2}, b\right)}{\beta(T)}\right]^{2} h^{2}, b\right)\right. \\
& +i\left(\frac{i}{q}\right)^{1 / 2}\left(\left[v-\frac{\left(v h^{2}, b\right)}{\beta(T)}\right] h, a\right) \\
& \left.-\frac{i}{2 q \beta(T)}\left(q\left(\frac{i}{q}\right)^{1 / 2} \alpha(T)-\left(v h^{2}, b\right)\right)^{2}\right\} d \sigma(v) \\
& =\int_{L_{a, b}^{2}[0, T]} \exp \left\{-\frac{i}{2 q}\left(v^{2} h^{2}, b\right)+i\left(\frac{i}{q}\right)^{1 / 2}(v h, a)\right\} d \sigma(v) \\
& =E^{a n f_{q}}[F]
\end{aligned}
$$

which concludes the proof of Theorem 4.3.

5. The generalized integral equation. Throughout this section we will choose $a(t)$, the mean function of the generalized Brownian motion process $Y$, to be identically equal to zero. Since $a(t) \equiv 0$ on $[0, T]$, we will denote $L_{a, b}^{2}[0, T]$ by $L_{b}^{2}[0, T]$ and $C_{a, b}[0, T]$ by $C_{b}[0, T]$. Let $y$ be the set of functions on $[0, T] \times \mathbb{R}$ of the form

$$
\theta(t, u)=\int_{\mathbb{R}} \exp \{i u v\} d v_{t}(v)
$$

where $\left\{v_{t}: 0 \leq t \leq T\right\}$ is a family from $M(\mathbb{R})$ with $\left\|v_{t}\right\| \in L_{b}[0, T]$, and for each $B \in$ $\mathscr{B}(\mathbb{R}), v_{t}(B)$ is a Borel measurable function of $t$. Let $\theta \in \mathcal{Y}$, let $F$ be given by

$$
F(x)=\exp \left\{\int_{0}^{T} \theta(t, x(t)) d t\right\}
$$

for s-a.e. $x \in C_{b}[0, T]$. Again it can be shown, as in [7], that the functions $\theta(t, u)$, $\int_{0}^{T} \theta(t, x(t)) d t$, and $F(x)$ are Borel measurable and that $\int_{0}^{T} \theta(t, x(t)) d t$ and $F(x)$ are elements of $S\left(L_{b}^{2}[0, T]\right)$. Hence there exists $\sigma=\sigma_{T} \in M\left(L_{b}^{2}[0, T]\right)$ such that

$$
F(x)=\int_{L_{b}^{2}[0, T]} \exp \left\{i \int_{0}^{T} v(t) d x(t)\right\} d \sigma(v)
$$

for s-a.e. $x \in C_{b}[0, T]$. Furthermore, the functional $G(x)=F(z(x, \cdot))$ with $h \in L_{\infty}[0, T]$, belongs to the Banach algebra $S\left(L_{b}^{2}[0, T]\right)[3]$. 
THEOREM 5.1. Let $\theta \in$ y be given by (5.1), let $F$ be given by (5.2), let $X$ be given by (4.1) with $h \in L_{\infty}[0, T]$, and for $(\eta, \lambda) \in \mathbb{R} \times(0, \infty)$, let

$$
H(T, \eta, \lambda)=\left(\frac{\lambda}{2 \pi \beta(T)}\right)^{1 / 2} \exp \left\{-\frac{\lambda \eta^{2}}{2 \beta(T)}\right\} E_{x}\left[F\left(\lambda^{-1 / 2} z(x, \cdot)\right) \mid \lambda^{-1 / 2} z(x, \cdot)\right](\eta) .
$$

Then for $(\eta, \lambda) \in \mathbb{R} \times(0, \infty)$, the function $H(T, \eta, \lambda)$ satisfies the generalized integral equation

$$
\begin{aligned}
& H(T, \eta, \lambda) \\
& =\left(\frac{\lambda}{2 \pi \beta(T)}\right)^{1 / 2} \exp \left\{-\frac{\lambda \eta^{2}}{2 \beta(T)}\right\} \\
& \quad+\int_{0}^{T} \int_{\mathbb{R}} \theta(s, \zeta) H(s, \zeta, \lambda) \cdot\left(\frac{\lambda}{2 \pi(\beta(T)-\beta(s))}\right)^{1 / 2} \exp \left\{-\frac{\lambda(\eta-\zeta)^{2}}{2(\beta(T)-\beta(s))}\right\} d \zeta d s .
\end{aligned}
$$

Proof. Let $(\eta, \lambda) \in \mathbb{R} \times(0, \infty)$ be given. By differentiating the function $\exp \left\{\int_{0}^{s} \theta\left(u, \lambda^{-1 / 2} z(x, u)\right) d u\right\}$ with respect to $s$ and then integrating the derivative on $[0, T]$, we obtain

$$
\begin{aligned}
\exp \left\{\int_{0}^{T} \theta\left(s, \lambda^{-1 / 2} z(x, s)\right) d s\right\} & \\
= & 1+\int_{0}^{T} \exp \left\{\int_{0}^{s} \theta\left(u, \lambda^{-1 / 2} z(x, u)\right) d u\right\} \theta\left(s, \lambda^{-1 / 2} z(x, s)\right) d s .
\end{aligned}
$$

Since the left-hand side of (5.6) is $\mu$-integrable, it follows that the second term of the right-hand side of (5.6) is $\mu$-integrable. Hence taking conditional expectations, and using (2.17), (2.19), and the Fubini theorem, we have

$$
\begin{gathered}
E_{x}\left[F\left(\lambda^{-1 / 2} z(x, \cdot)\right) \mid \lambda^{-1 / 2} z(x, \cdot)\right](\eta) \\
=1+E_{x}\left[\int_{0}^{T} \theta\left(s, \lambda^{-1 / 2} z(x, s)\right) \exp \left\{\int_{0}^{s} \theta\left(u, \lambda^{-1 / 2} z(x, u)\right) d u\right\} d s \mid \lambda^{-1 / 2} z(x, T)=\eta\right] \\
=1+E_{x}\left[\int_{0}^{T} \theta\left(s, \lambda^{-1 / 2}\left(z(x, s)-\frac{\beta(s)}{\beta(T)} z(x, T)\right)+\frac{\beta(s)}{\beta(T)} \eta\right)\right. \\
\left.\cdot \exp \left\{\int_{0}^{s} \theta\left(u, \lambda^{-1 / 2}\left(z(x, u)-\frac{\beta(u)}{\beta(T)} z(x, T)\right)+\frac{\beta(u)}{\beta(T)} \eta\right) d u\right\} d s\right] \\
=1+\int_{0}^{T} E_{x}\left[\theta\left(s, \lambda^{-1 / 2}\left(z(x, s)-\frac{\beta(s)}{\beta(T)} z(x, T)\right)+\frac{\beta(s)}{\beta(T)} \eta\right)\right. \\
\cdot \exp \left\{\int _ { 0 } ^ { s } \theta \left(u, \lambda^{-1 / 2}\left(z(x, u)-\frac{\beta(u)}{\beta(s)} z(x, s)\right)\right.\right. \\
\left.\left.\left.+\frac{\beta(u)}{\beta(s)}\left[\lambda^{-1 / 2}\left(z(x, s)-\frac{\beta(s)}{\beta(T)} z(x, T)\right)+\frac{\beta(s)}{\beta(T)} \eta\right]\right) d u\right\}\right] d s .
\end{gathered}
$$


Observe that the random variable $\lambda^{-1 / 2}(z(x, s)-\beta(s) z(x, T) / \beta(T))+\beta(s) \eta / \beta(T)$ is Gaussian with mean $\beta(s) \eta / \beta(T)$ and variance $\sigma^{2} \equiv \lambda^{-1}\left(\beta(s)-\beta^{2}(s) / \beta(T)\right)$ for $0 \leq$ $s<T$. Moreover, for $0 \leq u<s<T$, the two Gaussian random variables $\lambda^{-1 / 2}(z(x, s)-$ $\beta(s) z(x, T) / \beta(T))$ and $\lambda^{-1 / 2}(z(x, u)-\beta(u) z(x, s) / \beta(s))$ are independent. By applying these facts and the change of variable theorem to the last expression in (5.7), we have

$$
\begin{gathered}
E_{x}\left[F\left(\lambda^{-1 / 2} z(x, \cdot)\right) \mid \lambda^{-1 / 2} z(x, \cdot)\right](\eta) \\
=1+\int_{0}^{T} \int_{\mathbb{R}} E_{x}\left[\exp \left\{\int_{0}^{s} \theta\left(u, \lambda^{-1 / 2}\left(z(x, u)-\frac{\beta(u)}{\beta(s)} z(x, s)\right)+\frac{\beta(u)}{\beta(s)} \zeta\right) d u\right\}\right] \\
\cdot \theta(s, \zeta)\left(2 \pi \sigma^{2}\right)^{-1 / 2} \exp \left\{-\frac{(\zeta-\beta(s) \eta / \beta(T))^{2}}{2 \sigma^{2}}\right\} d \zeta d s \\
=1+\int_{0}^{T} \int_{\mathbb{R}}\left(2 \pi \sigma^{2}\right)^{-1 / 2} \theta(s, \zeta) E_{x}\left[\exp \left\{\int_{0}^{s} \theta\left(u, \lambda^{-1 / 2} z(x, u)\right) d u\right\} \mid \lambda^{-1 / 2} z(x, s)=\zeta\right] \\
\cdot \exp \left\{-\frac{(\zeta-\beta(s) \eta / \beta(T))^{2}}{2 \sigma^{2}}\right\} d \zeta d s .
\end{gathered}
$$

But we observe that

$$
\begin{aligned}
& \left(\frac{\lambda}{2 \pi \beta(T)}\right)^{1 / 2} \exp \left\{-\frac{\lambda \eta^{2}}{2 \beta(T)}\right\}\left(2 \pi \sigma^{2}\right)^{-1 / 2} \exp \left\{-\frac{(\zeta-\beta(s) \eta / \beta(T))^{2}}{2 \sigma^{2}}\right\} \\
& \cdot\left(\frac{2 \pi \beta(s)}{\lambda}\right)^{1 / 2} \exp \left\{\frac{\lambda \zeta^{2}}{2 \beta(s)}\right\}=\left(\frac{\lambda}{2 \pi(\beta(T)-\beta(s))}\right)^{1 / 2} \exp \left\{-\frac{\lambda(\eta-\zeta)^{2}}{2(\beta(T)-\beta(s))}\right\} .
\end{aligned}
$$

Hence by (5.8) and (5.9), we obtain

$$
\begin{aligned}
& H(T, \eta, \lambda)=\left(\frac{\lambda}{2 \pi \beta(T)}\right)^{1 / 2} \exp \left\{-\frac{\lambda \eta^{2}}{2 \beta(T)}\right\} \\
&+\int_{0}^{T} \int_{\mathbb{R}} \theta(s, \zeta)\left(\frac{\lambda}{2 \pi \beta(T)}\right)^{1 / 2} \exp \left\{-\frac{\lambda \eta^{2}}{2 \beta(T)}\right\}\left(2 \pi \sigma^{2}\right)^{-1 / 2} \\
& \cdot \exp \left\{-\frac{(\zeta-\beta(s) \eta / \beta(T))^{2}}{2 \sigma^{2}}\right\} \\
& \cdot E_{x}\left[\exp \left\{\int_{0}^{s} \theta\left(u, \lambda^{-1 / 2} z(x, u)\right) d u\right\} \mid \lambda^{-1 / 2} z(x, s)=\zeta\right] d \zeta d s \\
&= \\
&+\int_{0}^{T} \int_{\mathbb{R}} \theta(s, \zeta) H(s, \zeta, \lambda)\left(\frac{\lambda(T)}{2 \pi(\beta(T)-\beta(s))}\right)^{1 / 2} \\
& \cdot \exp \left\{-\frac{\lambda \eta^{2}}{2 \beta(T)}\right\} \\
& \cdot \exp \left\{-\frac{\lambda(\eta-\zeta)^{2}}{2(\beta(T)-\beta(s))}\right\} d \zeta d s,
\end{aligned}
$$

which completes the proof of the theorem. 
THEOREM 5.2. Let $F$ and $X$ be as in Theorem 5.1. Then the function

$$
H(T, \eta, \lambda)=\left(\frac{\lambda}{2 \pi \beta(T)}\right)^{1 / 2} \exp \left\{-\frac{\lambda \eta^{2}}{2 \beta(T)}\right\} E^{a n_{\lambda}}[F \mid X](\eta)
$$

satisfies the integral equation (5.5) for $(\eta, \lambda) \in \mathbb{R} \times \mathbb{C}_{+}$.

Proof. Since $H(T, \eta, \lambda)$ given by (5.11) satisfies the integral equation for $\lambda>0$, it suffices to show that both sides of (5.5) are analytic functions of $\lambda$ throughout $\mathbb{C}_{+}$. By Theorem $4.1, H(T, \eta, \lambda)$ exists and is analytic throughout $\mathbb{C}_{+}$. Hence it suffices to show that the second term on the right-hand side of (5.5), denoted by $h(\lambda)$, is an analytic function of $\lambda$ on $\mathbb{C}_{+}$. First an application of the dominated convergence theorem shows that $h(\lambda)$ is continuous on $\mathbb{C}_{+}$; an appropriate dominating function is obtained as in the following argument and so will be omitted here. Let $\Delta$ be a triangular path in $\mathbb{C}_{+}$. We need only show that $\int_{\Delta} h(\lambda) d \lambda=0$. If we can apply the Fubini theorem to the integral on $\Delta \times[0, T] \times \mathbb{R}$, this will be clear by the Cauchy integral theorem. Let $D=\sup \{|\lambda|: \lambda \in \Delta\}$ and $E=\inf \{\operatorname{Re} \lambda: \lambda \in \Delta\}$. Then

$$
\begin{aligned}
\left(\frac{D}{E}\right)^{1 / 2} & \left(\frac{E}{2 \pi(\beta(T)-\beta(s))}\right)^{1 / 2}\left\|v_{s}\right\|\left(\frac{D}{2 \pi \beta(T)}\right)^{1 / 2} \\
& \cdot \exp \left\{-\frac{E}{2 \beta(T)} \eta^{2}\right\}\|\sigma\| \exp \left\{-\frac{E}{2(\beta(T)-\beta(s))}(\eta-\zeta)^{2}\right\}
\end{aligned}
$$

is a dominating function integrable with respect to $(s, \zeta, \lambda)$ on $[0, T] \times \mathbb{R} \times \Delta$.

ThEOREM 5.3. Let $F$ and $X$ be as in Theorem 5.1. Then for $(\eta, q) \in \mathbb{R} \times(\mathbb{R}-\{0\})$, the function

$$
H(T, \eta,-i q)=\left(\frac{q}{2 \pi i \beta(T)}\right)^{1 / 2} \exp \left\{\frac{i q \eta^{2}}{2 \beta(T)}\right\} E^{a n f_{q}[F \mid X](\eta)}
$$

satisfies the generalized integral equation

$$
\begin{aligned}
H(T, \eta,-i q)= & \left(\frac{q}{2 \pi i \beta(T)}\right)^{1 / 2} \exp \left\{\frac{i q \eta^{2}}{2 \beta(T)}\right\} \\
& +\int_{0}^{T}\left(\frac{q}{2 \pi i(\beta(T)-\beta(s))}\right)^{1 / 2} \int_{\mathbb{R}} \theta(s, \zeta) H(s, \zeta,-i q) \\
& \cdot \exp \left\{\frac{i q(\eta-\zeta)^{2}}{2(\beta(T)-\beta(s))}\right\} d \zeta d s .
\end{aligned}
$$

Proof. We note that the techniques used in Theorem 5.2 will not work here since $\lim _{\lambda \rightarrow-i q}(|\lambda| / \operatorname{Re} \lambda)=+\infty$. By Theorem 4.1, we have

$$
\lim _{\lambda \rightarrow-i q} H(T, \eta, \lambda)=H(T, \eta,-i q) .
$$

Next let

$$
G(s, \zeta, \lambda)=\left(\frac{\lambda}{2 \pi(\beta(T)-\beta(s))}\right)^{1 / 2} \theta(s, \zeta) H(s, \zeta, \lambda) \exp \left\{-\frac{\lambda(\eta-\zeta)^{2}}{2(\beta(T)-\beta(s))}\right\}
$$


for $s \in(0, T), \zeta \in \mathbb{R}$ and $\lambda \neq 0$ with $\operatorname{Re} \lambda \geq 0$. Since the integral equation (5.5) holds for all $\lambda \in \mathbb{C}_{+}$, it suffices to show that

$$
\lim _{\lambda \rightarrow-i q} \int_{0}^{T} \int_{\mathbb{R}} G(s, \zeta, \lambda) d \zeta d s=\int_{0}^{T} \int_{\mathbb{R}} G(s, \zeta,-i q) d \zeta d s .
$$

Using the dominated convergence theorem in steps 3, 4, and 6 below, we obtain the equation

$$
\begin{aligned}
\int_{0}^{T} \int_{\mathbb{R}} G(s, \zeta,-i q) d \zeta d s & =\int_{0}^{T} \lim _{A \rightarrow+\infty} \int_{\mathbb{R}} G(s, \zeta,-i q) \exp \left\{-\frac{\zeta^{2}}{2 A}\right\} d \zeta d s \\
& =\int_{0}^{T} \lim _{A \rightarrow+\infty} \int_{\mathbb{R}} \lim _{\lambda \rightarrow-i q} G(s, \zeta, \lambda) \exp \left\{-\frac{\zeta^{2}}{2 A}\right\} d \zeta d s \\
& =\int_{0}^{T} \lim _{A \rightarrow+\infty} \lim _{\lambda \rightarrow-i q} \int_{\mathbb{R}} G(s, \zeta, \lambda) \exp \left\{-\frac{\zeta^{2}}{2 A}\right\} d \zeta d s \\
& =\int_{0}^{T} \lim _{\lambda \rightarrow-i q} \lim _{A \rightarrow+\infty} \int_{\mathbb{R}} G(s, \zeta, \lambda) \exp \left\{-\frac{\zeta^{2}}{2 A}\right\} d \zeta d s \\
& =\int_{0}^{T} \lim _{\lambda \rightarrow-i q} \int_{\mathbb{R}} G(s, \zeta, \lambda) d \zeta d s \\
& =\lim _{\lambda \rightarrow-i q} \int_{0}^{T} \int_{\mathbb{R}} G(s, \zeta, \lambda) d \zeta d s .
\end{aligned}
$$

In fact, we will find a function that dominates the function

$$
L(s)=\int_{\mathbb{R}} G(s, \zeta, \lambda) \exp \left\{-\frac{\zeta^{2}}{2 A}\right\} d \zeta
$$

for all $A$ sufficiently large, for all $\lambda \in \mathbb{C}_{+}$sufficiently close to $-i q$, and that is in $L^{1}[0, T]$ as a function of $s$. In particular, we will find a dominating function that is independent of $A$ and dominates for all $\lambda \neq 0$ such that $\operatorname{Re} \lambda \geq 0$ and $|\lambda| \leq \lambda_{0}=2|q|+1$. Also it suffices to take the limit as $\lambda \rightarrow-i q$ along a horizontal line since we know that the limit in (5.17) exists.

By using the definitions of $L(s)$ and $G(s, \zeta, \lambda)$, and then (4.2), (5.1), (5.3), and (5.11), we have

$$
\begin{aligned}
L(s)= & \left(\frac{\lambda}{2 \pi(\beta(T)-\beta(s))}\right)^{1 / 2}\left(\frac{\lambda}{2 \pi \beta(s)}\right)^{1 / 2} \\
& \cdot \int_{\mathbb{R}}\left[\int_{\mathbb{R}} \exp \{i u \zeta\} d v_{s}(u)\right] \exp \left\{-\frac{\lambda(\eta-\zeta)^{2}}{2(\beta(T)-\beta(s))}-\frac{\lambda \zeta^{2}}{2 \beta(s)}-\frac{\zeta^{2}}{2 A}\right\} \\
& \cdot \int_{L_{b}^{2}[0, s]} \exp \left\{\frac{i \zeta}{\beta(s)}\left(v h^{2}, b\right)_{s}-\frac{1}{2 \lambda}\left(v^{2} h^{2}, b\right)_{s}+\frac{1}{2 \lambda \beta(s)}\left(v h^{2}, b\right)_{s}^{2}\right\} d \sigma_{s}(v) d \zeta
\end{aligned}
$$

where $\sigma_{s} \in M\left(L_{b}^{2}[0, s]\right)$ and $\left(v h^{2}, b\right)_{s}=\int_{0}^{s} v(w) h^{2}(w) d b(w)$.

Now we can apply the Fubini theorem to the integral in $L(s)$. First carrying out the integration with respect to $\zeta$, we obtain 


$$
\begin{aligned}
& L(s)=\left(\frac{\lambda}{2 \pi \beta(T)}\right)^{1 / 2}(\left.\frac{A \lambda \beta(T)}{\beta(s)(\beta(T)-\beta(s))+A \lambda \beta(T)}\right)^{1 / 2} \\
& \cdot \int_{\mathbb{R}} \int_{L_{b}^{2}[0, s]} \exp \left\{-\frac{1}{2 \lambda}\left(v^{2} h^{2}, b\right)_{s}+\frac{1}{2 \lambda \beta(s)}\left(v h^{2}, b\right)_{s}^{2}\right. \\
&- \frac{\lambda \eta^{2}}{(\beta(T)-\beta(s))}-\frac{A \beta(s)(\beta(T)-\beta(s))}{2(A \lambda \beta(T)+\beta(s)(\beta(T)-\beta(s)))} \\
&\left.\cdot\left[u+\frac{1}{\beta(s)}\left(v h^{2}, b\right)_{s}-\frac{i \lambda \eta}{\beta(T)-\beta(s)}\right]^{2}\right\} d \sigma_{s}(v) d v_{s}(u) .
\end{aligned}
$$

Now we claim that for all $A>0$ and all $\lambda \neq 0$ such that $\operatorname{Re} \lambda \geq 0$ and $|\lambda| \leq \lambda_{0}=2|q|+1$,

$$
|L(s)| \leq\left(\frac{\lambda_{0}}{2 \pi \beta(T)}\right)^{1 / 2}\left\|\sigma_{s}\right\|\left\|v_{s}\right\| \leq\left(\frac{\lambda_{0}}{2 \pi \beta(T)}\right)^{1 / 2}\|\sigma\|\left\|v_{s}\right\| .
$$

Once this claim is established, the proof is complete because the expression on the right-hand side of (5.22) is in $L^{1}[0, T]$ as a function of $s$. To obtain the inequality (5.22) we use the following results:

(i) For all $\operatorname{Re} \lambda \geq 0$ and $A>0,|A \lambda \beta(T) /(\beta(s)(\beta(T)-\beta(s))+A \lambda \beta(T))| \leq 1$ since $\beta(\cdot)$ is a strictly increasing function with $\beta(0)=0$.

(ii) $\left|\exp \left\{-\left(v^{2} h^{2}, b\right)_{s} /(2 \lambda \beta(s))+\left(v h^{2}, b\right)_{s}^{2} /(2 \lambda \beta(s))\right\}\right| \leq 1$ since $\operatorname{Re}(-1 / 2 \lambda \beta(s)) \leq$ 0 and $\left(v h^{2}, b\right)_{s}^{2} \leq \beta(s)\left(v^{2} h^{2}, b\right)_{s}$ by the Cauchy-Schwarz inequality.

(iii) Formula (5.22) will follow once we show that

$$
\begin{aligned}
\mid \exp \left\{-\frac{\lambda \eta^{2}}{2(\beta(T)-\beta(s))}-\frac{A \beta(s)(\beta(T)-\beta(s))}{2(A \lambda \beta(T)+\beta(s)(\beta(T)-\beta(s)))}\right. \\
\left.\cdot\left[u+\frac{1}{\beta(s)}\left(v h^{2}, b\right)_{s}-\frac{i \lambda \eta}{\beta(T)-\beta(s)}\right]^{2}\right\} \mid \leq 1
\end{aligned}
$$

for all appropriate values of the variables involved. To show (iii), it suffices to show that the real part of the exponent is nonpositive. Recalling that $\lambda=p-i q$ and letting

$$
y=u+\frac{1}{\beta(s)}\left(v h^{2}, b\right)_{s}-\frac{q \eta}{(\beta(T)-\beta(s))},
$$

we obtain that

$$
\begin{aligned}
\operatorname{Re}\{ & -\frac{\lambda}{2(\beta(T)-\beta(s))} \eta^{2} \\
& \left.-\frac{A \beta(s)(\beta(T)-\beta(s))}{2(A \lambda \beta(T)+\beta(s)(\beta(T)-\beta(s)))}\left[u+\frac{1}{\beta(s)}\left(v h^{2}, b\right)_{s}-\frac{i \lambda \eta}{\beta(T)-\beta(s)}\right]^{2}\right\} \\
= & -\frac{A \beta(s)(\beta(T)-\beta(s))[A p \beta(T)+\beta(T)(\beta(T)-\beta(s))]}{2\left[(A p \beta(T)+\beta(s)(\beta(T)-\beta(s)))^{2}+(A q \beta(T))^{2}\right]} \\
& \cdot\left[y+\frac{A p q \beta(T) \eta}{(\beta(T)-\beta(s))[A p \beta(T)+\beta(s)(\beta(T)-b(s))]}\right]^{2}-\frac{p \eta^{2}}{2(\beta(T)-\beta(s))} \\
& \cdot\left[1-\frac{A^{3} p q^{2} \beta(s) \beta^{2}(T)+A p \beta(s)[A p \beta(T)+\beta(s)(\beta(T)-\beta(s))]^{2}}{\left[(A p \beta(T)+\beta(s)(\beta(T)-\beta(s)))^{2}+(A q \beta(T))^{2}\right][A p \beta(T)+\beta(s)(\beta(T)-\beta(s))]}\right] .
\end{aligned}
$$


But for $p \geq 0$ and $0 \leq s \leq T$, we see that

$$
\begin{aligned}
& A^{3} p q^{2} \beta(s) \beta^{2}(T)+A p \beta(s)[A p \beta(T)+\beta(s)(\beta(T)-\beta(s))]^{2} \\
& \quad \leq\left[(A p \beta(T)+\beta(s)(\beta(T)-\beta(s)))^{2}+(A q \beta(T))^{2}\right][A p \beta(T)+\beta(s)(\beta(T)-\beta(s))]
\end{aligned}
$$

and hence

$$
1-\frac{A^{3} p q^{2} \beta(s) \beta^{2}(T)+A p \beta(s)[A p \beta(T)+\beta(s)(\beta(T)-\beta(s))]^{2}}{\left[(A p \beta(T)+\beta(s)(\beta(T)-\beta(s)))^{2}+(A q \beta(T))^{2}\right][A p \beta(T)+\beta(s)(\beta(T)-\beta(s))]} \geq 0 .
$$

Thus the expression on the left-hand side of (5.25) is non-positive which completes the proof of Theorem 5.3.

THEOREM 5.4. Let $F$ and $X$ be as in Theorem 5.1. Let $\psi$ be given by

$$
\psi(\eta)=\int_{\mathbb{R}} \exp \{i u \eta\} d \phi(u)
$$

for some $\phi \in M(\mathbb{R})$, and let

$$
G(x)=F(x) \psi(x(T)+\eta) .
$$

Then for all real $q \neq 0$, we have that

$$
\begin{aligned}
& \Gamma(T, \eta, q) \equiv E^{a n f_{a}[G]} \\
&=\int_{L_{b}^{2}[0, T]}\left[\exp \left\{-\frac{i}{2 q}\left(\left[v-\frac{\left(v h^{2}, b\right)}{\beta(T)}\right]^{2} h^{2}, b\right)\right\}\right. \\
&\left.\cdot \int_{\mathbb{R}} \exp \left\{i u \eta-\frac{i \beta(T)}{2 q}\left(u+\frac{\left(v h^{2}, b\right)}{\beta(T)}\right)^{2}\right\} d \phi(u)\right] d \sigma(v) .
\end{aligned}
$$

In addition, we have the alternative expression

$$
E^{a n f_{q}}[G]=\bar{\int}_{\mathbb{R}} E^{a n f_{q}}[F \mid X](\zeta-\eta)\left(\frac{q}{2 \pi i \beta(T)}\right)^{1 / 2} \exp \left\{\frac{i q(\zeta-\eta)^{2}}{2 \beta(T)}\right\} \psi(\zeta) d \zeta
$$

where

$$
E^{a n f_{a}}[F \mid X](\zeta-\eta)=\int_{L_{b}^{2}[0, T]} \exp \left\{\frac{i(\zeta-\eta)}{\beta(T)}\left(v h^{2}, b\right)-\frac{i}{2 q}\left(\left[v-\frac{\left(v h^{2}, b\right)}{\beta(T)}\right]^{2} h^{2}, b\right)\right\} d \sigma(v) .
$$

Proof. We will first obtain the expression (5.30). Proceeding as in the proof of Theorem 4.3, and then using (5.28) and (4.2), we have that for all $\lambda>0$, 


$$
\begin{aligned}
& E^{a n_{\lambda}[G]=} \int_{\mathbb{R}} E_{x}\left[F\left(\lambda^{-1 / 2} z(x, \cdot)\right) \psi\left(\lambda^{-1 / 2} z(x, T)+\eta\right) \mid \lambda^{-1 / 2} z(x, T)+\eta\right](\zeta) \\
& \cdot\left(\frac{\lambda}{2 \pi \beta(T)}\right)^{1 / 2} \exp \left\{-\frac{\lambda(\zeta-\eta)^{2}}{2 \beta(T)}\right\} d \zeta \\
&=\int_{\mathbb{R}} E_{x}\left[F\left(\lambda^{-1 / 2} z(x, \cdot)\right) \mid \lambda^{-1 / 2} z(x, T)\right](\zeta-\eta) \\
& \cdot \psi(\zeta)\left(\frac{\lambda}{2 \pi \beta(T)}\right)^{1 / 2} \exp \left\{-\frac{\lambda(\zeta-\eta)^{2}}{2 \beta(T)}\right\} d \zeta \\
&= \int_{\mathbb{R}} \int_{L_{b}^{2}[0, T]} \exp \left\{\frac{i(\zeta-\eta)}{\beta(T)}\left(v h^{2}, b\right)-\frac{1}{2 \lambda}\left(\left[v-\frac{\left(v h^{2}, b\right)}{\beta(T)}\right]^{2} h^{2}, b\right)\right\} d \sigma(v) \\
& \cdot\left(\frac{\lambda}{2 \pi \beta(T)}\right)^{1 / 2} \exp \left\{-\frac{\lambda(\zeta-\eta)^{2}}{2 \beta(T)}\right\} \int_{\mathbb{R}} \exp \{i u \zeta\} d \phi(u) d \zeta .
\end{aligned}
$$

We next use the Fubini theorem, and then carry out the integration with respect to $\zeta$ and obtain the formula

$$
\begin{aligned}
E^{a n_{\lambda}[G]=\int_{L_{b}^{2}[0, T]}}[ & \exp \left\{-\frac{1}{2 \lambda}\left(\left[v-\frac{\left(v h^{2}, b\right)}{\beta(T)}\right]^{2} h^{2}, b\right)\right\} \\
& \left.\cdot \int_{\mathbb{R}} \exp \left\{i u \eta-\frac{\beta(T)}{2 \lambda}\left(u+\frac{\left(v h^{2}, b\right)}{\beta(T)}\right)^{2}\right\} d \phi(u)\right] d \sigma(v) .
\end{aligned}
$$

But clearly the right-hand side of (5.34) is analytic in $\lambda$ on $\mathbb{C}_{+}$and continuous for $\lambda$ in $\tilde{\mathbb{C}}_{+}$, and hence $E^{a n f_{a}}[G]$ exists and given by (5.30). To obtain equation (5.31), we use (4.7), (5.32) and the Fubini theorem:

$$
\begin{aligned}
& \int_{\mathbb{R}} E_{x}^{a n f_{q}}[F \mid X](\zeta-\eta)\left(\frac{q}{2 \pi i \beta(T)}\right)^{1 / 2} \exp \left\{\frac{i q(\zeta-\eta)^{2}}{2 \beta(T)}\right\} \psi(\zeta) d \zeta \\
& =\lim _{A \rightarrow+\infty} \int_{\mathbb{R}} E^{a n f_{q}}[F \mid X](\zeta-\eta)\left(\frac{q}{2 \pi i \beta(T)}\right)^{1 / 2} \exp \left\{\frac{i q(\zeta-\eta)^{2}}{2 \beta(T)}-\frac{\zeta^{2}}{2 A}\right\} \psi(\zeta) d \zeta \\
& =\lim _{A \rightarrow+\infty} \int_{L_{b}^{2}[0, T]} \int_{\mathbb{R}}\left(\frac{q}{2 \pi i \beta(T)}\right)^{1 / 2} \exp \left\{-\frac{i}{2 q}\left(\left[v-\frac{\left(v h^{2}, b\right)}{\beta(T)}\right]^{2} h^{2}, b\right)\right\} \\
& \cdot \exp \left\{\frac{i(\zeta-\eta)}{\beta(T)}\left(v h^{2}, b\right)+\frac{i q(\zeta-\eta)^{2}}{2 \beta(T)}-\frac{\zeta^{2}}{2 A}\right\} \\
& \cdot \int_{\mathbb{R}} \exp \{i u \zeta\} d \phi(u) d \zeta d \sigma(v) \\
& =\lim _{A \rightarrow+\infty} \int_{L_{b}^{2}[0, T]}\left[\exp \left\{-\frac{i}{2 q}\left(\left[v-\frac{\left(v h^{2}, b\right)}{\beta(T)}\right]^{2} h^{2}, b\right)-\frac{i \eta}{\beta(T)}\left(v h^{2}, b\right)\right\}\right. \\
& \cdot \int_{\mathbb{R}}\left(\frac{q}{2 \pi i \beta(T)}\right)^{1 / 2}\left(\frac{2 \pi A \beta(T)}{\beta(T)-i q A}\right)^{1 / 2} \\
& \left.\cdot \exp \left\{\frac{i q \eta^{2}}{2 \beta(T)}-\frac{A\left[q \eta-\left(\left(v h^{2}, b\right)+\beta(T) u\right)\right]^{2}}{2 \beta(T)(\beta(T)-i q A)}\right\} d \phi(u)\right] d \sigma(v)
\end{aligned}
$$




$$
\begin{aligned}
& =\int_{L_{b}^{2}[0, T]}\left[\exp \left\{-\frac{i}{2 q}\left(\left[v-\frac{\left(v h^{2}, b\right)}{\beta(T)}\right]^{2} h^{2}, b\right)\right\}\right. \\
& \left.\qquad \int_{\mathbb{R}} \exp \left\{i u \eta-\frac{i \beta(T)}{2 q}\left(u+\frac{\left(v h^{2}, b\right)}{\beta(T)}\right)^{2}\right\} d \phi(u)\right] d \sigma(v) \\
& =E^{a n f_{q}[G] .}
\end{aligned}
$$

REMARK 5.5. (i) For $\theta \in$ y given by (5.1), $F$ given by (5.2), $G$ given by (5.29), and using Theorem 5.3 and (5.31) and proceeding as in the proof of [7, Theorem 7.1], we observe that $\Gamma(T, \eta, q)$ is a solution of the generalized Schrödinger integral equation

$$
\begin{aligned}
& \Gamma(T, \eta, q)=\left(\frac{q}{2 \pi i \beta(T)}\right)^{1 / 2} \int_{\mathbb{R}} \psi(\zeta) \exp \left\{\frac{i q(\eta-\zeta)^{2}}{2 \beta(T)}\right\} d \zeta \\
& +\int_{0}^{T}\left(\frac{q}{2 \pi i(\beta(T)-\beta(s))}\right)^{1 / 2} \int_{\mathbb{R}} \theta(s, \zeta) \Gamma(s, \zeta, q) \exp \left\{\frac{i q(\eta-\zeta)^{2}}{2(\beta(T)-\beta(s))}\right\} d \zeta d s .
\end{aligned}
$$

(ii) We also note that (5.31) can rewritten as

$$
\Gamma(T, \eta, q)=H(T,-(\cdot),-q i) * \psi(\eta)
$$

where $*$ denotes convolution and $H(T, \eta,-q i)$ is given by (5.13).

ACKNOWLEDGEMENT. The first author was supported by KOSEF Post-Doctoral Fellowship in Korea in 1997 and the second author was supported by BSRI, 98-1412. The first author wishes to express his gratitude to the University of Nebraska-Lincoln for its hospitality.

\section{REFERENCES}

[1] R. H. Cameron and D. A. Storvick, Some Banach algebras of analytic Feynman integrable functionals, Analytic functions, Kozubnik 1979 (Proc. Seventh Conf., Kozubnik, 1979) (Berlin), Lecture Notes in Mathematics, vol. 798, Springer, 1980, pp. 18-67. MR 83f:46059. Zbl 439.28007.

[2] D. M. Chung, Scale-invariant measurability in abstract Wiener spaces, Pacific J. Math. 130 (1987), no. 1, 27-40. MR 88m:28009. Zbl 634.28007.

[3] D. M. Chung, C. Park, and D. Skoug, Generalized Feynman integrals via conditional Feynman integrals, Michigan Math. J. 40 (1993), no. 2, 377-391. MR 94h:28008. Zbl 799.60049.

[4] D. M. Chung and D. L. Skoug, Conditional analytic Feynman integrals and a related Schrödinger integral equation, SIAM J. Math. Anal. 20 (1989), no. 4, 950-965. MR 90k:28028. Zbl 678.28007.

[5] G. W. Johnson and D. L. Skoug, Scale-invariant measurability in Wiener space, Pacific J. Math. 83 (1979), no. 1, 157-176. MR 81b:28016. Zbl 414.60066.

[6] _ Notes on the Feynman integral. II, J. Funct. Anal. 41 (1981), no. 3, 277-289. MR 85k:81070b. Zbl 459.28012.

[7] _ Notes on the Feynman integral. III. The Schröedinger equation, Pacific J. Math. 105 (1983), no. 2, 321-358. MR 85k:81070c. Zbl 505.28010.

[8] C. Park and D. Skoug, A Kac-Feynman integral equation for conditional Wiener integrals, J. Integral Equations Appl. 3 (1991), no. 3, 411-427. MR 93i:28011. Zbl 751.45003. 
[9] J. Yeh, Stochastic Processes and the Wiener Integral, Marcel Dekker Inc., New York, 1973. MR 57\#14166. Zbl 277.60018.

Chang: Department of Mathematics, Dankook University, Cheonan 330-714, Korea E-mail address: sejchang@anseo.dankook.ac.kr

Kang: Department of Mathematical Education, Chonnam national University, KWANGJU 500, KOREA

E-mail address: kangsj@chonnam.chonnam.ac.kr

SKOUG: DePARTMENT OF MATHEMATICS AND STATISTICS, UNIVERSITY OF NEBRASKA-LINCOLN, LINCOLN NE, 68588-0323, USA

E-mail address: dskoug@un 1 info.un 1 .edu 


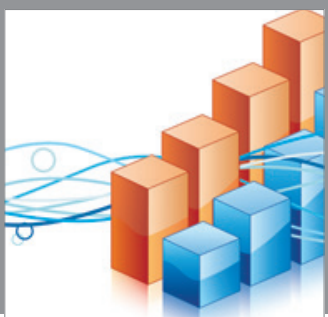

Advances in

Operations Research

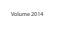

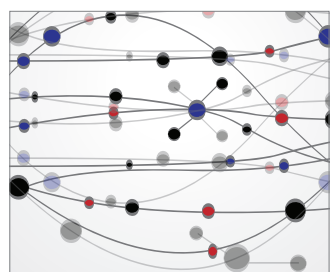

\section{The Scientific} World Journal
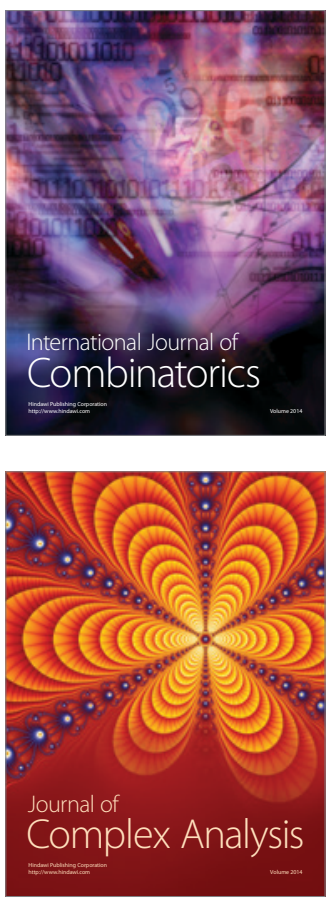

International Journal of

Mathematics and

Mathematical

Sciences
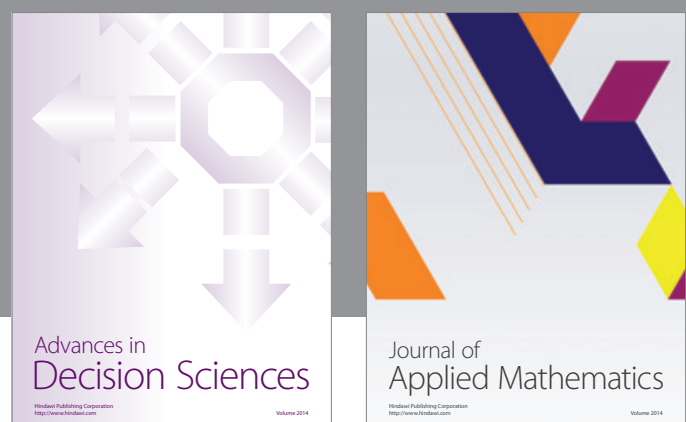

Journal of

Applied Mathematics
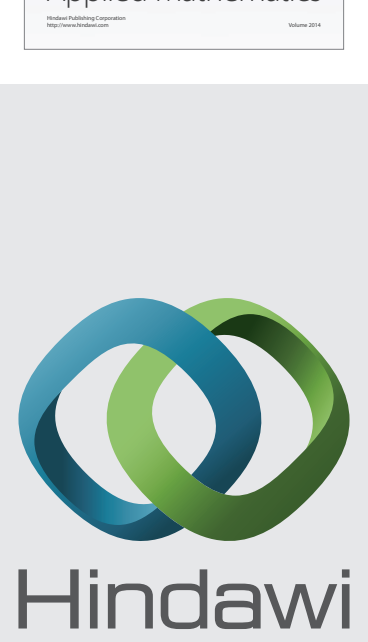

Submit your manuscripts at http://www.hindawi.com
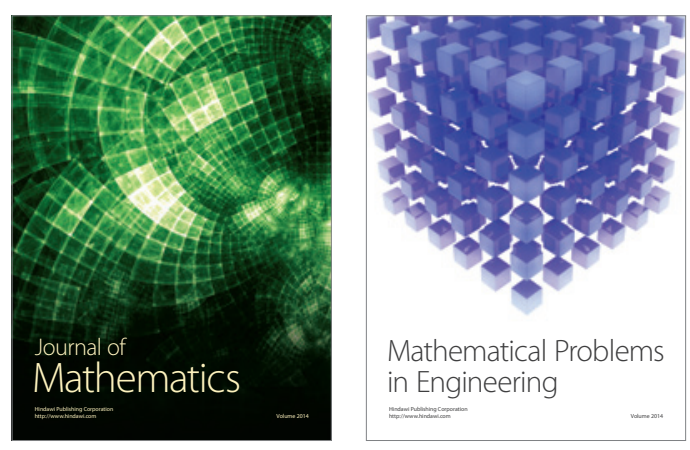

Mathematical Problems in Engineering
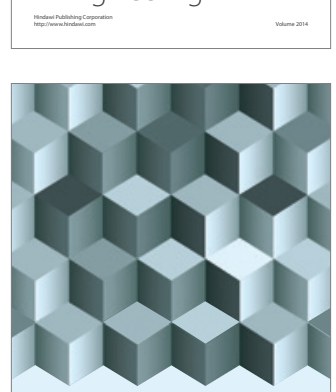

Journal of

Function Spaces
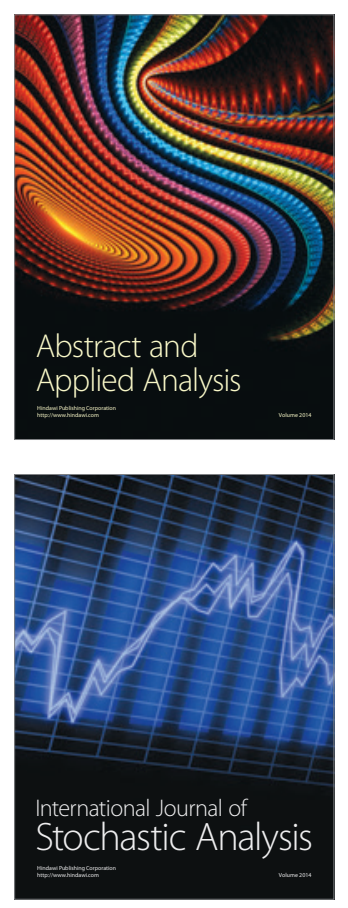

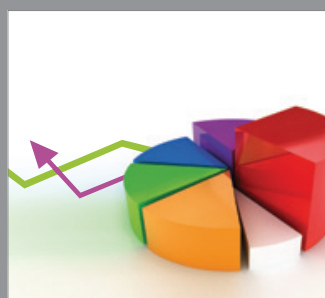

ournal of

Probability and Statistics

Promensencen
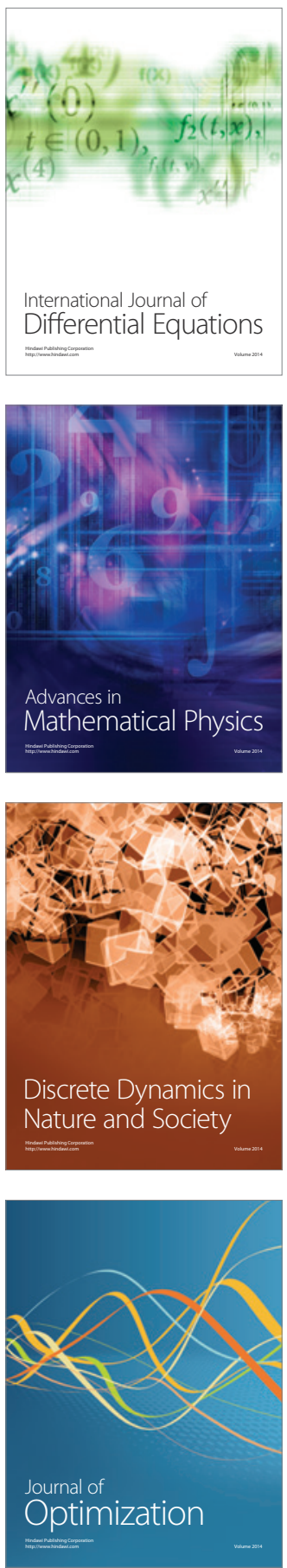